\title{
Integrated analysis of the differential cellular and EBV miRNA expression profiles in microdissected nasopharyngeal carcinoma and non-cancerous nasopharyngeal tissues
}

\author{
XUN-XUN WAN ${ }^{1,2}$, HONG YI ${ }^{1}$, JIA-QUAN QU ${ }^{1}$, QIU-YAN HE ${ }^{1}$ and ZHI-QIANG XIAO ${ }^{1}$ \\ ${ }^{1}$ Research Center of Carcinogenesis and Targeted Therapy, Xiangya Hospital, Central South University, Changsha, \\ Hunan 410008; ${ }^{2}$ Department of Biochemistry and Molecular Biology, College of Medicine, \\ Hunan Normal University, Changsha, Hunan 410006, P.R. China
}

Received May 13, 2015; Accepted June 26, 2015

DOI: 10.3892/or.2015.4237

\begin{abstract}
Nasopharyngeal carcinoma (NPC) is commonly diagnosed in southern Asia. MicroRNAs (miRNAs) are small non-coding RNAs that regulate gene expression post-transcriptionally. Increasing evidence suggests that the dysregulation of miRNAs promotes NPC tumorigenesis. Epstein-Barr virus (EBV) infection and EBV-encoded miRNAs are also associated with the development of NPC. However, it is unclear how cellular and EBV miRNAs jointly regulate target genes and signaling pathways in NPC. In the present study, we analyzed the differential cellular and EBV miRNA expression profiles in 20 pooled NPC tissues using microarrays. We found that 19 cellular miRNAs and 9 EBV miRNAs were upregulated and 31 cellular miRNAs were downregulated in NPC tissues. Gene Ontology (GO) and the Kyoto Encyclopedia of Genes and Genomes (KEGG) pathway analysis indicated that the 19 upregulated miRNAs target mainly the p53 signaling pathway in cancer, whereas the downregulated miRNAs regulate pathways related to cancer, focal adhesion and Erb, and MAPK signaling. In contrast, the upregulated EBV miRNAs target primarily the TGF- $\beta$ and Wnt signaling pathways. Data also suggested that cellular miR-34b, miR-34c, miR-18a, miR-200a/b, miR-449a, miR-31 and let-7 may be dysregulated in NPCs, and that the aberrant activation of their target genes in the p53 pathway and cell cycle enhance NPC cell survival
\end{abstract}

Correspondence to: Professor Zhi-Qiang Xiao, Research Center of Carcinogenesis and Targeted Therapy, Xiangya Hospital, Central South University, Changsha, Hunan 410008, P.R. China

E-mail: zqxiao2001@hotmail.com

Abbreviations: NPC, nasopharyngeal carcinoma; NNET, normal nasopharyngeal epithelial tissue; EBV, Epstein-Barr virus; GO, Gene Ontology; KEGG, Kyoto Encyclopedia of Genes and Genomes; LCM, laser capture microdissected; NCBI-GEO, National Center for Biotechnology Information-Gene Expression Omnibus; qRT-PCR, quantitative reverse transcription-PCR

Key words: nasopharyngeal carcinoma, microarray, Epstein-Barr virus, miRNA and proliferation. In addition, EBV-miRNAs such as BART3 and BART5 target genes in the p53, TGF- $\beta$ and Wnt signaling pathways to modulate NPC apoptosis and transformation. To better elucidate the interaction between miRNAs and target genes, we constructed an anti-correlated cellular and EBV miRNA/target gene regulatory network. The current findings may help dissect the roles played by cellular and EBV miRNAs during NPC tumorigenesis, and also provide useful biomarkers for the diagnosis and treatment of NPCs.

\section{Introduction}

Nasopharyngeal carcinoma (NPC) is a human malignancy derived from the epithelium of the nasopharyngeal recess. It has a particularly high incidence in Southeast Asia, and is associated with genetic and epigenetic events, as well as Epstein-Barr virus (EBV) infection. It is important to elucidate the molecular mechanisms of NPC carcinogenesis.

MicroRNAs (miRNAs) are short non-coding RNA molecules and are involved in post-transcriptional regulation of genes involved in a variety of biological functions, including cellular development, differentiation, proliferation and apoptosis (1-3). The dysregulation of miRNAs appears to play a crucial role in cancer, where they exert effects as oncogenes or tumor suppressors (4). Approximately $50 \%$ of miRNAs are localized in cancer-associated genomic regions, and their expression can be altered by genomic amplification, loss of heterozygosity, viral integration and genomic rearrangements (5). Large-scale profiling studies revealed a global alteration of the miRNA expression patterns in human types of cancers (6-8), and distinct miRNA expression signatures have been proposed as diagnostic and prognostic markers for various types of human cancer $(9,10)$.

A growing number of cellular miRNAs are now known to be involved in NPC via targeting specific mRNAs. Most of these, including miR-29c, miR-218, miR-26a, miR-663, let-7, miR-141, miR-216b and miR-200a, play an important role in the onset and progression of NPC (11-18). A unique feature of NPC is its strong association with EBV latent infection. The full-length EBV genome is detected in all cases of NPC, suggesting that products of the EBV genome are involved 
in the pathogenesis of this malignancy $(19,20)$. As well as EBV-encoded protein-coding genes, latently infected NPC cells and tissues also express high levels of viral miRNAs (21). EBV is the first human virus known to express miRNAs. To date, $>44 \mathrm{EBV}$ miRNAs have been discovered in EBV-positive cell lines, and they exhibit different expression patterns depending on the sample and stage of infection (21). Since EBV severely dysregulates the mRNA profiles of host cells (22), EBV miRNAs may have oncogenic properties. Functional studies revealed that several EBV-encoded miRNAs play roles in NPC by targeting a variety of cellular (23-26) and viral (27-30) mRNAs. Therefore, both cellular and EBV miRNAs play a crucial role in NPC (31).

Various genome-wide miRNA expression profiling studies using microarray-based approaches yielded abundant information regarding the phenotypes of cancers. Distinct patterns of cellular miRNA expression and specific miRNA signatures in NPC have been identified, which are associated with clinicopathological characteristics as well as prognosis, suggesting that miRNAs play key roles in the development, invasion and metastasis of NPC (32-36). Several studies have performed EBV miRNA expression profiling in NPC cells and tissues (21,37-39). However, there has been no systematic analysis of both differentially expressed cellular and EBV miRNA expression profiles in NPC tissues.

In the present study, we used microarray analyses to compare the differences in the cellular and EBV miRNA expression profiles in pooled laser capture microdissected (LCM) NPC tissues and normal nasopharyngeal epithelial tissues (NNETs). We identified the target genes of differential cellular and EBV miRNAs using database predictions, and comparing the resulting information with the gene expression in NPC reported in the Gene Expression Omnibus (GEO) (GSE34573 and GSE12452). In addition, we analyzed the functions and pathways modulated by the cellular and EBV miRNA target genes. Finally, we constructed an anticorrelated cellular and EBV miRNA/target gene regulatory network. The resulting data may be helpful for elucidating the roles of cellular and EBV miRNA deregulation in NPC carcinogenesis, and also revealing the global trends in cellular and EBV miRNAs and gene interactions in NPC.

\section{Materials and methods}

Sample collection, laser capture microdissection and total RNA extraction. Paired NPC tissues and adjacent NNETs from 20 patients with confirmed NPC were obtained from the Tumor Hospital of Hunan Province (China) at the time of diagnosis before any treatment, and were used for microarrays and quantitative reverse transcription-PCR (qRT-PCR). Tissue specimens were obtained using fiber optic nasopharyngoscopy directly at the tumor growth site, and the adjacent side with an observed normal mucosal morphology was used for the corresponding non-tumor pair. All patients signed an informed consent form for participation in the present study, which had been previously reviewed by the Institutional Review Board. All tissue samples were verified by histopathology before microdissection. Laser capture microdissection (LCM) was used to purify the target cells from the fresh NPC tissues and NNETs using a Leica AS LMD system (Leica, Mannheim,
Germany), as previously described (40). The cells obtained from LCM were collected in the cap of a $0.5-\mathrm{ml}$ Eppendorf (EP) tube containing TRIzol reagent (Invitrogen, Life Technologies, Shanghai, China). Total RNA was extracted using TRIzol according to the manufacturer's instructions. The concentration and integrity of the resulting RNA were evaluated using an Agilent 2100 Bioanalyzer (Agilent Technologies, Santa Clara, CA, USA). Only total RNA samples with an RNA integrity number (RIN) $\geq 7$ were used for miRNA microarray analyses and qRT-PCR. To diminish the effect of biological sample variation on the results of the microarray analysis, equal amounts of total RNA from the microdissected cells of 10 different individuals were pooled to generate one common sample for each tissue type.

miRNA microarrays. RNA samples were labeled using a miRCURY Hy3/Hy5 labeling kit and hybridized on the miRCURY locked nucleic acid (LNA) array (version 10.0; Exiqon, KangChen Biotech, Shanghai, China) according to the manufacturer's instructions. The array contains probes for 850 human miRNAs and $39 \mathrm{EBV}$ miRNAs, and uses a one-color technique. The arrays were scanned using a laser scanner (GenePix 4000B; Molecular Devices, LLC, Sunnyvale, CA, USA), and the raw data were normalized and analyzed using image analysis software (GenePix Pro v6.0) to generate a median value for each miRNA from the repeated probes. The miRNA signal intensities were $\log ^{2}$ transformed, and differentially expressed miRNAs were analyzed using the significance analysis of microarrays (SAM, version 3.01). P-values were calculated using t-tests, and differentially detected miRNAs with a $\geq 2.0$ - or $\leq 0.50$ fold-change and a P-value $<0.01$ were considered to be statistically significant.

Quantitative RT-PCR. To validate the miRNA expression levels determined using microarrays, stem-looped qRT-PCR was performed to detect the expression of 15 cellular miRNAs (hsa-miR-200b, hsa-miR-34c-5p, hsa-miR-31, hsa-miR-34b*, hsa-miR-656, hsa-miR-374a, hsa-miR-150, hsa-miR-200a, hsa-miR-365-1, hsa-miR-15a, hsa-miR-20a, hsa-miR-17, hsa-miR-18a, hsa-miR-106a and hsa-miR-412) and five EBV miRNAs (ebv-miR-BART5, ebv-miR-BART6-3p, ebv-miR-BART3, ebv-miR-BART1-5p and ebv-miR-BART7) in an independent set of samples consisting of 10 pairs of LCM-purified NPCs and NNETs. Briefly, $2 \mu \mathrm{g}$ of total RNA was reverse transcribed into cDNA using a reverse transcription kit according to the manufacturer's protocol (Promega, Madison, WI, USA). miRNA-specific primers (Bulge-Loop ${ }^{\mathrm{TM}}$ miRNA qPCR primers) for the differentially expressed miRNAs were synthesized by GeneChem Co. (Shanghai, China) and are summarized in Table I. The RT products were amplified using real-time PCR with the miScript SYBR-Green PCR kit (Qiagen, Shanghai, China) according to the manufacturer's instructions; U6 was used as the internal control. The following PCR program was used: $95^{\circ} \mathrm{C}$ for $5 \mathrm{~min}$, followed by 40 cycles of $95^{\circ} \mathrm{C}$ for $10 \mathrm{sec}, 60^{\circ} \mathrm{C}$ for $20 \mathrm{sec}$ and $78^{\circ} \mathrm{C}$ for $20 \mathrm{sec}$. The real-time PCR primers used to amplify the miRNAs were designed based on the miRNA sequences provided by the Sanger Center miRNA Registry and synthesized by GeneChem; their sequences are provided in Table II. All qRT-PCR reactions were performed in triplicate and 
Table I. The specific stem-looped RT primers to representatives of differentially expressed miRNAs.

\begin{tabular}{|c|c|}
\hline miRNA & RT primers \\
\hline hsa-miR-200b & 5'-GTCGTATCCAGTGCGTGTCGTGGAGTCGGCAATTGCACTGGATACGACTCATCAT-3' \\
\hline hsa-miR-34c-5p & 5'-GTCGTATCCAGTGCGTGTCGTGGAGTCGGCAATTGCACTGGATACGACGCAATC-3' \\
\hline hsa-miR-31 & 5'-GTCGTATCCAGTGCGTGTCGTGGAGTCGGCAATTGCACTGGATACGACCAGCTAT-3' \\
\hline hsa-miR-34b* & 5'-GTCGTATCCAGTGCGTGTCGTGGAGTCGGCAATTGCACTGGATACGACCAATCA-3' \\
\hline hsa-miR-656 & 5'-GTCGTATCCAGTGCGTGTCGTGGAGTCGGCAATTGCACTGGATACGACAGAGGTT-3' \\
\hline hsa-miR-374a & 5'-GTCGTATCCAGTGCGTGTCGTGGAGTCGGCAATTGCACTGGATACGACCACTTAT-3' \\
\hline hsa-miR-200a & 5'-GTCGTATCCAGTGCGTGTCGTGGAGTCGGCAATTGCACTGGATACGACACATCGT-3' \\
\hline hsa-miR-150 & 5'-GTCGTATCCAGTGCGTGTCGTGGAGTCGGCAATTGCACTGGATACGACCACTGGT-3' \\
\hline hsa-miR-365-1 & 5'-GTCGTATCCAGTGCGTGTCGTGGAGTCGGCAATTGCACTGGATACGACATAAGGA-3' \\
\hline hsa-miR-15a & 5'-GTCGTATCCAGTGCGTGTCGTGGAGTCGGCAATTGCACTGGATACGACCACAAAC-3' \\
\hline hsa-miR-412 & 5'-GTCGTATCCAGTGCGTGTCGTGGAGTCGGCAATTGCACTGGATACGACACGGCT-3' \\
\hline hsa-miR-20a & 5'-GTCGTATCCAGTGCGTGTCGTGGAGTCGGCAATTGCACTGGATACGACCTACCTG-3' \\
\hline hsa-miR-18a & 5'-GTCGTATCCAGTGCGTGTCGTGGAGTCGGCAATTGCACTGGATACGACCTATCTG-3' \\
\hline hsa-miR-17 & 5'-GTCGTATCCAGTGCGTGTCGTGGAGTCGGCAATTGCACTGGATACGACCTACCTG-3' \\
\hline hsa-miR-106a & 5'-GTCGTATCCAGTGCGTGTCGTGGAGTCGGCAATTGCACTGGATACGACCTACCTG-3' \\
\hline ebv-miR-BART7 & 5'-GTCGTATCCAGTGCGTGTCGTGGAGTCGGCAATTGCACTGGATACGACCCCTGG-3' \\
\hline ebv-miR-BART1-5p & 5'-GTCGTATCCAGTGCGTGTCGTGGAGTCGGCAATTGCACTGGATACGACCACAGCA-3' \\
\hline ebv-miR-BART3 & 5'-GTCGTATCCAGTGCGTGTCGTGGAGTCGGCAATTGCACTGGATACGACACACCT-3' \\
\hline ebv-miR-BART6-3p & 5'-GTCGTATCCAGTGCGTGTCGTGGAGTCGGCAATTGCACTGGATACGACTCTAAGG-3' \\
\hline ebv-miR-BART5 & 5'-GTCGTATCCAGTGCGTGTCGTGGAGTCGGCAATTGCACTGGATACGACCGATGGG-3' \\
\hline U6 & 5'-CGCTTCACGAATTTGCGTGTCAT-3' \\
\hline
\end{tabular}

RT, reverse transcription.

products were quantified using the $2^{-\Delta \Delta \mathrm{Ct}}$ method normalized against U6. All qRT-PCR reactions were performed on an ABI GeneAmp PCR System 9700 (ABI, Carlsbad, CA, USA).

Identification of cellular and EBV miRNA target genes. The putative target genes of the cellular miRNAs expressed differentially in miRNA microarrays were predicted using three databases (miRanda, PITA and TargetScan, 2013). The cellular miRNA target genes in any of the three databases were selected, and their potential differential gene expression profiles (GSE34573 and GSE12452) in NPC biopsies from the National Center for Biotechnology Information (NCBI)-Gene Expression Omnibus (GEO) were compared. Potential target genes, which were differentially expressed in the gene expression profiles in NPC and also had a negative correlation with the cellular miRNA expression in the microarrays, were identified and further analyzed.

The putative host target genes of the differentially expressed EBV miRNAs in the microarrays were predicted using three databases (RepTar (41), DIANA-microT v3.0 (42) and starBase (43), 2013). The host target genes recorded in any of these databases were selected and their potential differential gene expression profiles (GSE34573 and GSE12452) in NPC biopsies from the NCBI-GEO were compared. The predicted host target genes that were correlated negatively with the EBV miRNA expression profiles were identified and further analyzed. The putative viral target genes of the differentially expressed EBV miRNAs in the microarrays were predicted using the vHoT database (2013) (44), which predicts the viral target genes of virus-derived miRNAs using popular miRNA target prediction algorithms including TargetScan, miRanda and PITA. The targets of the differentially expressed EBV miRNAs in the viral genome were identified according to the match sites of these miRNAs in the EBV genome and the following criteria: a total context score 0 (TargetScan), a minimum free energy (MFE) $\leq 0 \mathrm{kcal} / \mathrm{mol}$ (miRanda) and a ddG threshold $\leq 10$ (PITA).

GO and Kyoto Encyclopedia of Genes and Genomes (KEGG) pathway analysis of the cellular and EBV miRNA target genes. To understand the functions of the predicted host and virus target genes of the cellular and EBV miRNAs, we performed ontology classification and KEGG pathway analysis using DAVID Bioinformatics Resources 6.7 (http://david. abcc.ncifcrf.gov/home.jsp). The target genes were assigned to functional groups based on biological processes. Correlation was defined as significantly enriched when $\mathrm{P} \leq 0.05$.

Integrative analysis of the cellular miRNA and gene expression profiles to construct an miRNA/gene regulatory network. The post-transcriptional regulatory network of an miRNA and gene is defined as a directed, bipartite graph in which the expression of the miRNA/target gene interacting pairs are anti-correlated. The differentially expressed miRNAs and genes of interest were determined using pathways extracted from KEGG as the primary nodal networks. The networks were drawn using Cytoscape 2.8.3. The resulting post-transcriptional network identified the genes regulated by the same 
Table II. The real-time PCR primers for amplification of the mature miRNAs.

\begin{tabular}{|c|c|c|c|c|}
\hline miRNA & & Bidirectional primers & $\begin{array}{c}\text { Annealing } \\
\text { temperature }\left({ }^{\circ} \mathrm{C}\right)\end{array}$ & $\begin{array}{c}\text { Product } \\
\text { length (bp) }\end{array}$ \\
\hline \multirow{2}{*}{ hsa-miR-200b } & GSP & 5'-GGGGTAATACTGCCTGGT-3' & & \\
\hline & $\mathrm{R}$ & 5'-TGCGTGTCGTGGAGTC-3' & 60 & 63 \\
\hline \multirow{2}{*}{ hsa-miR-34c-5p } & GSP & 5'-GGGAGGCAGTGTAGTTAGC-3' & & \\
\hline & $\mathrm{R}$ & 5'-CAGTGCGTGTCGTGGAGT-3' & 60 & 66 \\
\hline \multirow{2}{*}{ hsa-miR-31 } & GSP & 5'-GGAGGCAAGATGCTGGC-3' & & \\
\hline & $\mathrm{R}$ & 5'-CAGTGCGTGTCGTGGAGT-3' & 60 & 64 \\
\hline \multirow[t]{2}{*}{ hsa-miR-34b* } & GSP & 5'-GGGTAGGCAGTGTCATTAGC-3' & & \\
\hline & $\mathrm{R}$ & 5'-CAGTGCGTGTCGTGGAGT-3' & 60 & 66 \\
\hline \multirow[t]{2}{*}{ hsa-miR-656 } & GSP & 5'-GGCGGAATATTATACAGTCAA-3' & & \\
\hline & $\mathrm{R}$ & 5'-CAGTGCGTGTCGTGGAGT-3' & 60 & 66 \\
\hline \multirow[t]{2}{*}{ hsa-miR-374a } & GSP & 5'-GGCACCTTATAATACAACCTG-3' & & \\
\hline & $\mathrm{R}$ & 5'-TGCGTGTCGTGGAGTC-3' & 60 & 65 \\
\hline \multirow[t]{2}{*}{ hsa-miR-200a } & GSP & 5'-GGGGTAACACTGTCTGGTAG-3' & & \\
\hline & $\mathrm{R}$ & 5'-TGCGTGTCGTGGAGTC-3' & 60 & 63 \\
\hline \multirow[t]{2}{*}{ hsa-miR-150 } & GSP & 5'-GCTCTCCCAACCCTTGT-3' & & \\
\hline & $\mathrm{R}$ & 5'-TGCGTGTCGTGGAGTC-3' & 60 & 61 \\
\hline \multirow[t]{2}{*}{ hsa-miR-365-1 } & GSP & 5'-GCACTTCACCTGGTCCACT-3' & & \\
\hline & $\mathrm{R}$ & 5'-CAGTGCGTGTCGTGGAGT-3' & 60 & 65 \\
\hline \multirow[t]{2}{*}{ hsa-miR-15a } & GSP & 5'-GGGTAGCAGCACATAATGG-3' & & \\
\hline & $\mathrm{R}$ & 5'-CAGTGCGTGTCGTGGAGT-3' & 60 & 67 \\
\hline \multirow{2}{*}{ hsa-miR-412 } & GSP & 5'-GCACTTCACCTGGTCCACT-3' & & \\
\hline & $\mathrm{R}$ & 5'-CAGTGCGTGTCGTGGAGT-3' & 60 & 65 \\
\hline \multirow[t]{2}{*}{ hsa-miR-20a } & GSP & 5'-GGGTAAAGTGCTTATAGTGC-3' & & \\
\hline & $\mathrm{R}$ & 5'-TGCGTGTCGTGGAGTC-3' & 60 & 63 \\
\hline \multirow[t]{2}{*}{ hsa-miR-18a } & GSP & 5'-GGGTAAGGTGCATCTAGTGC-3' & & \\
\hline & $\mathrm{R}$ & 5'-TGCGTGTCGTGGAGTC-3' & 60 & 64 \\
\hline \multirow{2}{*}{ hsa-miR-17 } & GSP & 5'-GGGCAAAGTGCTTACAGTGC-3' & & \\
\hline & $\mathrm{R}$ & 5'-TGCGTGTCGTGGAGTC-3' & 60 & 65 \\
\hline \multirow[t]{2}{*}{ hsa-miR-106a } & GSP & 5'-GGGGAAAAGTGCTTACAGTG-3' & & \\
\hline & $\mathrm{R}$ & 5'-CAGTGCGTGTCGTGGAGT-3' & 60 & 67 \\
\hline \multirow[t]{2}{*}{ ebv-miR-BART7 } & GSP & 5'-GGGCATCATAGTCCAGTGT-3' & & \\
\hline & $\mathrm{R}$ & 5'-CAGTGCGTGTCGTGGAGT-3' & 60 & 65 \\
\hline \multirow[t]{2}{*}{ ebv-miR-BART1-5p } & GSP & 5'-GGCTCTTAGTGGAAGTGACG-3' & & \\
\hline & $\mathrm{R}$ & 5'-CAGTGCGTGTCGTGGAGT-3' & 60 & 67 \\
\hline \multirow[t]{2}{*}{ ebv-miR-BART3 } & GSP & 5'-GGCGCACCACTAGTCACC-3' & & \\
\hline & $\mathrm{R}$ & 5'-CAGTGCGTGTCGTGGAGT-3' & 60 & 64 \\
\hline \multirow[t]{2}{*}{ ebv-miR-BART6-3p } & GSP & 5'-AAGCGGGGATCGGACTA-3' & & \\
\hline & $\mathrm{R}$ & 5'-CAGTGCGTGTCGTGGAGT-3' & 60 & 65 \\
\hline \multirow[t]{2}{*}{ ebv-miR-BART5 } & GSP & 5'-CGGCAAGGTGAATATAGC-3' & & \\
\hline & $\mathrm{R}$ & 5'-TGCGTGTCGTGGAGTC-3' & 60 & 64 \\
\hline \multirow[t]{2}{*}{ U6 } & $\mathrm{F}$ & 5'-GCTTCGGCAGCACATATACTAAAAT-3' & & \\
\hline & $\mathrm{R}$ & 5'-CGCTTCACGAATTTGCGTGTCAT-3' & 60 & 89 \\
\hline
\end{tabular}

GSP are specific primers corresponding to the miRNA. R are primters matching with RT primers.

miRNAs, as well as miRNAs that regulate the specific genes of functional relevance.
Establishing an intercross regulatory network of cellular and EBV miRNAs in NPC. To understand the interaction 

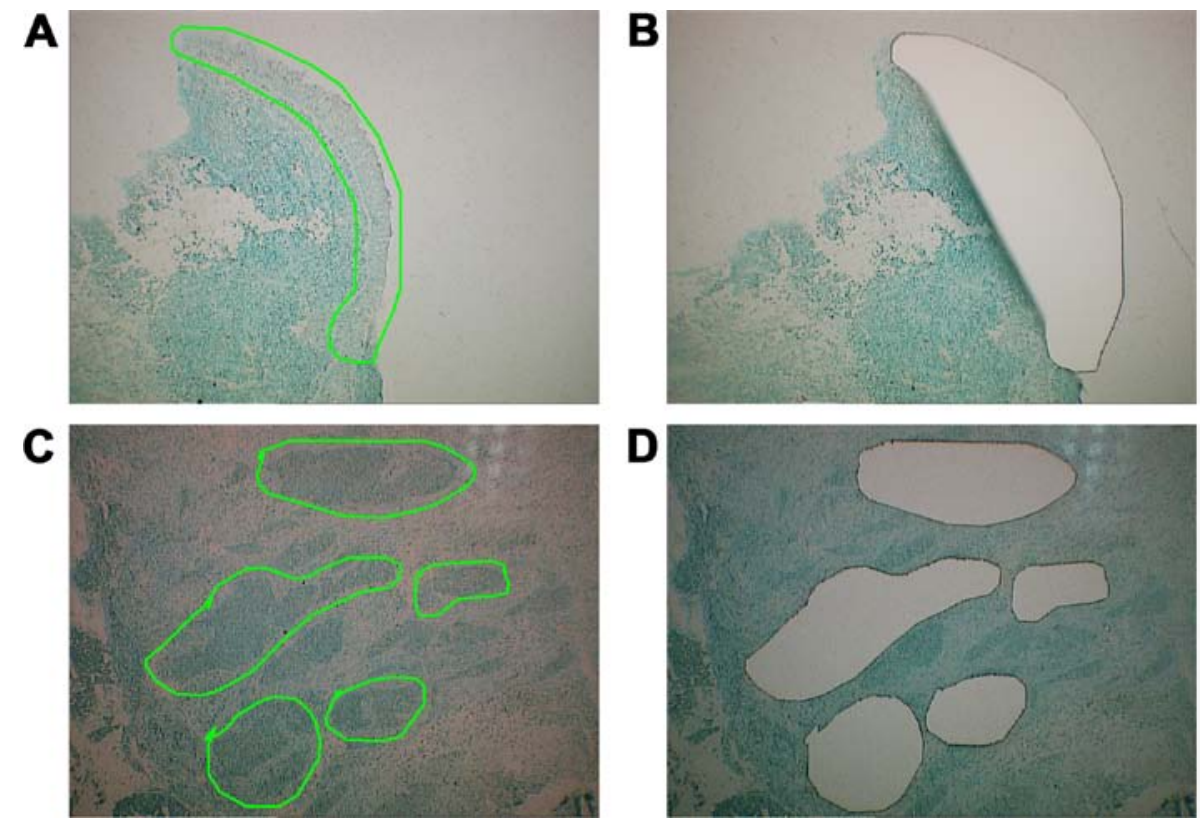

Figure 1. Microdissection of nasopharyngeal carcinoma (NPC) and normal nasopharyngeal epithelial tissues (NNETs). Eight-micrometer-thick frozen sections of undifferentiated nasopharyngeal carcinoma (WHO type III) and adjacent NNETs were fixed in 75\% alcohol and stained with $0.5 \%$ violet-free methyl green. All solutions for staining were supplemented with RNAase inhibitor. Following staining, the sections were microdissected immediately using a Leica AS LMD system. NNET before (A) and after (B) microdissection. NPC tissues before (C) and after (D) microdissection. Original magnification, x100.

between cellular and EBV miRNAs, we identified the target genes regulated by both cellular and viral miRNAs that were anti-correlated with the miRNA expression observed in the microarrays. We then established a gene regulatory network that was co-regulated by both cellular and viral miRNAs in NPC. The networks were drawn using Cytoscape 2.8.3.

\section{Results}

Differentially expressed cellular and EBV miRNAs in NPC tissues and NNETs. miRNA expression profiling was performed using pooled LCM-purified NPC tissues and paired NNETs (Fig. 1), and miRCURY LNA arrays (version 10.0) containing 850 human and 39 EBV miRNAs. miRNAs were scored as positive using SAM; differential expression with a $\geq 2$ fold-change was regarded as significant. These analyses identified 50 cellular miRNAs and 9 differential EBV miRNAs that were expressed differentially between NPC and normal tissues. Among these, 19 cellular miRNAs and 9 EBV miRNAs were upregulated, whereas 31 cellular miRNAs were downregulated in the NPC tissues compared with the NNETs (Table III). Importantly, miR-106a, miR-142-3p, miR-155, miR-17, miR-18a, let-7, miR-200a, miR-200b, miR-31, miR-34b, miR-34c and miR449a were previously reported to be differentially regulated $(11,32,33,36,39)$.

Compared with all previous published studies, several novel miRNAs that were upregulated in NPC were identified in the present study: hsa-miR-142-5p, hsa-miR-193a-3p, hsa-miR-221*, hsa-miR-30b*, hsa-miR30c-2*, hsa-miR-338-5p, hsa-miR-412, hsa-miR-550 and hsa-miR-637. In addition several novel miRNAs that were downregulated in NPC were identified: hsa-miR-105*, hsamiR-133b, hsa-miR-138-1*, hsa-miR-200c*, hsa-miR-326, hsa-miR-328, hsa-miR-365, hsa-miR-374a, hsa-miR-450b-3p,
hsa-miR-506, hsa-miR-513-5p, hsa-miR-518a-3p, hsa-miR574-5p, hsa-miR-647, hsa-miR-656, hsa-miR-886-3p and hsa-miR-886-5p.

Validation of the miRNA microarray results using $q R T-P C R$. To validate the microarray data, qRT-PCR was performed to quantify the expression of 15 cellular miRNAs and $5 \mathrm{EBV}$ miRNAs in an independent set of 10 LCM-purified NPCs and paired NNETs. Data revealed that the expression of hsa-miR-200b, hsa-miR-34c-5p, hsa-miR-31, hsa-miR-34b*, hsa-miR-656, hsa-miR-374a, hsa-miR-150, hsa-miR-200a, hsa-miR-365-1 and hsa-miR-15a was significantly decreased, whereashsa-miR-20a,hsa-miR-17,hsa-miR-18a,hsa-miR-106a, hsa-miR-412, ebv-miR-BART5, ebv-miR-BART6-3p, ebvmiR-BART3, ebv-miR-BART1-5p and ebv-miR-BART7 were significantly increased in the NPC tissues when compared with the NNETs. These observations were consistent with the results of the miRNA microarrays (Fig. 2).

Prediction of the miRNA target genes. The putative target genes of 50 differentially expressed cellular miRNAs were predicted using the three databases as described in Materials and methods. A total of 69,869 target genes were recorded by the databases (data not shown). To identify more genuine target genes, all the predicted cellular miRNA targets were compared with the differential gene expression profiles in NPC tissues within NCBI-GEO. This identified 555 cellular miRNA target genes that were expressed differentially in NPC and negatively correlated with the expression of the differentially expressed miRNAs. Among these target genes, 218 and 337 were regulated by the upregulated and downregulated miRNAs, respectively (data not shown).

The putative host target genes of the nine upregulated EBV miRNAs were predicted using the three databases as 
Table III. Microarray-based detection of the differentially expressed miRNAs in the NPC tissues and the NNETs.

\begin{tabular}{|c|c|c|c|c|}
\hline \multirow[b]{2}{*}{ miRNA } & \multirow[b]{2}{*}{ Chromosome location } & \multicolumn{2}{|c|}{ Normalized value } & \multirow{2}{*}{$\begin{array}{l}\text { Fold-change } \\
\text { NPC/NNET }\end{array}$} \\
\hline & & NNET & NPC & \\
\hline \multicolumn{5}{|c|}{ Upregulated cellular miRNAs } \\
\hline hsa-miR-106a & $\mathrm{Xq} 26.2$ & 0.08 & 0.40 & 4.77 \\
\hline hsa-miR-142-3p & $17 \mathrm{q} 22$ & 0.20 & 0.40 & 2.00 \\
\hline hsa-miR-142-5p & $17 q 22$ & 0.11 & 0.22 & 2.00 \\
\hline hsa-miR-143 & $5 q 32$ & 0.05 & 0.15 & 3.22 \\
\hline hsa-miR-155 & $21 \mathrm{q} 21.3$ & 0.15 & 0.35 & 2.35 \\
\hline hsa-miR-16 & $13 q 14.2$ & 0.12 & 0.28 & 2.30 \\
\hline hsa-miR-17 & $13 q 31.3$ & 0.11 & 0.36 & 3.15 \\
\hline hsa-miR-18a & $13 q 31.3$ & 0.03 & 0.15 & 4.33 \\
\hline hsa-miR-193a-3p & $17 q 11.2$ & 0.03 & 0.13 & 4.13 \\
\hline hsa-miR-206 & $6 \mathrm{p} 12.2$ & 0.23 & 0.55 & 2.35 \\
\hline hsa-miR-20a & $13 q 31.3$ & 0.96 & 1.96 & 2.04 \\
\hline hsa-miR-21 & $17 \mathrm{q} 23.1$ & 0.28 & 0.58 & 2.04 \\
\hline hsa-miR-221* & Xp11.3 & 0.63 & 1.43 & 2.27 \\
\hline hsa-miR-30b* & $8 q 24.22$ & 5.32 & 14.14 & 2.66 \\
\hline hsa-miR-30c-2* & $6 q 13$ & 7.33 & 17.96 & 2.45 \\
\hline hsa-miR-338-5p & $17 \mathrm{q} 25.3$ & 0.18 & 0.92 & 5.17 \\
\hline hsa-miR-412 & $14 q 32.31$ & 0.36 & 1.14 & 3.16 \\
\hline hsa-miR-550 & $7 \mathrm{p} 14.3$ & 19.23 & 45.50 & 2.37 \\
\hline hsa-miR-637 & $19 \mathrm{p} 13.3$ & 0.17 & 0.36 & 2.06 \\
\hline \multicolumn{5}{|c|}{ Downregulated cellular miRNAs } \\
\hline hsa-let-7b* & $22 q 13.31$ & 1.26 & 0.04 & 0.04 \\
\hline hsa-miR-1 & $20 q 13.33$ & 2.00 & 1.03 & 0.51 \\
\hline hsa-miR-105* & $\mathrm{Xq} 28$ & 0.19 & 0.09 & 0.50 \\
\hline hsa-miR-133b & $6 \mathrm{p} 12.2$ & 3.69 & 0.03 & 0.01 \\
\hline hsa-miR-138-1* & $3 \mathrm{p} 21.32$ & 6.91 & 3.13 & 0.45 \\
\hline hsa-miR-150 & $19 q 13.33$ & 2.20 & 0.65 & 0.30 \\
\hline hsa-miR-15a & $13 q 14.2$ & 1.93 & 0.35 & 0.18 \\
\hline hsa-miR-15b & $3 \mathrm{q} 25.33$ & 1.98 & 0.16 & 0.08 \\
\hline hsa-miR-195 & $17 \mathrm{p} 13.1$ & 0.24 & 0.01 & 0.04 \\
\hline hsa-miR-200a & $1 \mathrm{p} 36.33$ & 0.35 & 0.17 & 0.47 \\
\hline hsa-miR-200b & $1 \mathrm{p} 36.33$ & 0.42 & 0.16 & 0.38 \\
\hline hsa-miR-200c* & $12 \mathrm{p} 13.31$ & 1.33 & 0.42 & 0.31 \\
\hline hsa-miR-31 & $9 p 21.3$ & 0.12 & 0.01 & 0.12 \\
\hline hsa-miR-326 & $11 \mathrm{q} 13.4$ & 0.87 & 0.14 & 0.16 \\
\hline hsa-miR-328 & $16 q 22.1$ & 0.29 & 0.14 & 0.49 \\
\hline hsa-miR-335 & $7 q 32.2$ & 0.15 & 0.04 & 0.23 \\
\hline hsa-miR-34b* & $11 q 23.1$ & 0.63 & 0.00 & 0.01 \\
\hline hsa-miR-34c-5p & $11 q 23.1$ & 1.81 & 0.10 & 0.06 \\
\hline hsa-miR-365 & $16 \mathrm{p} 13.12 / 17 \mathrm{q} 11.2$ & 1.51 & 0.25 & 0.16 \\
\hline hsa-miR-374a & $\mathrm{Xq} 13.2$ & 3.27 & 0.05 & 0.02 \\
\hline hsa-miR-449a & $5 q 11.2$ & 0.63 & 0.02 & 0.04 \\
\hline hsa-miR-450b-3p & $\mathrm{Xq} 26.3$ & 0.13 & 0.03 & 0.25 \\
\hline hsa-miR-486-5p & $8 p 11.21$ & 1.90 & 0.79 & 0.42 \\
\hline hsa-miR-506 & $\mathrm{Xq} 27.3$ & 0.36 & 0.14 & 0.38 \\
\hline hsa-miR-513-5p & $\mathrm{Xq} 27.3$ & 12.49 & 4.95 & 0.40 \\
\hline hsa-miR-518a-3p & $19 q 13.42$ & 5.23 & 2.68 & 0.51 \\
\hline hsa-miR-574-5p & $4 \mathrm{p} 14$ & 2.19 & 0.61 & 0.28 \\
\hline hsa-miR-647 & $20 \mathrm{q} 13.33$ & 0.86 & 0.37 & 0.43 \\
\hline hsa-miR-656 & $14 \mathrm{q} 32.31$ & 1.59 & 0.09 & 0.06 \\
\hline hsa-miR-886-3p & $5 q 31.1$ & 0.41 & 0.18 & 0.44 \\
\hline hsa-miR-886-5p & $5 q 31.1$ & 0.70 & 0.30 & 0.42 \\
\hline
\end{tabular}


Table III. Continued.

\begin{tabular}{|c|c|c|c|c|}
\hline \multirow[b]{2}{*}{ miRNA } & \multirow[b]{2}{*}{ Chromosome location } & \multicolumn{2}{|c|}{ Normalized value } & \multirow{2}{*}{$\begin{array}{l}\text { Fold-change } \\
\text { NPC/NNET }\end{array}$} \\
\hline & & NNET & NPC & \\
\hline \multicolumn{5}{|l|}{ Upregulated EBV miRNA } \\
\hline ebv-miR-BART8* & & 0.08 & 0.28 & 3.37 \\
\hline ebv-miR-BART4 & & 0.04 & 0.12 & 3.47 \\
\hline ebv-miR-BART5 & & 0.12 & 0.52 & 4.45 \\
\hline ebv-miR-BART1-5p & & 0.06 & 0.25 & 4.19 \\
\hline ebv-miR-BART18-3p & & 0.14 & 0.28 & 2.02 \\
\hline ebv-miR-BART10 & & 0.00 & 0.22 & 110.83 \\
\hline ebv-miR-BART6-3p & & 0.02 & 0.17 & 7.22 \\
\hline ebv-miR-BART3 & & 0.004 & 0.64 & 160.19 \\
\hline ebv-miR-BART7 & & 0.004 & 0.44 & 109.9 \\
\hline
\end{tabular}

EBV, Epstein-Barr virus; NNET normal nasopharyngeal epithelial tissue; NPC, nasopharyngeal carcinoma.
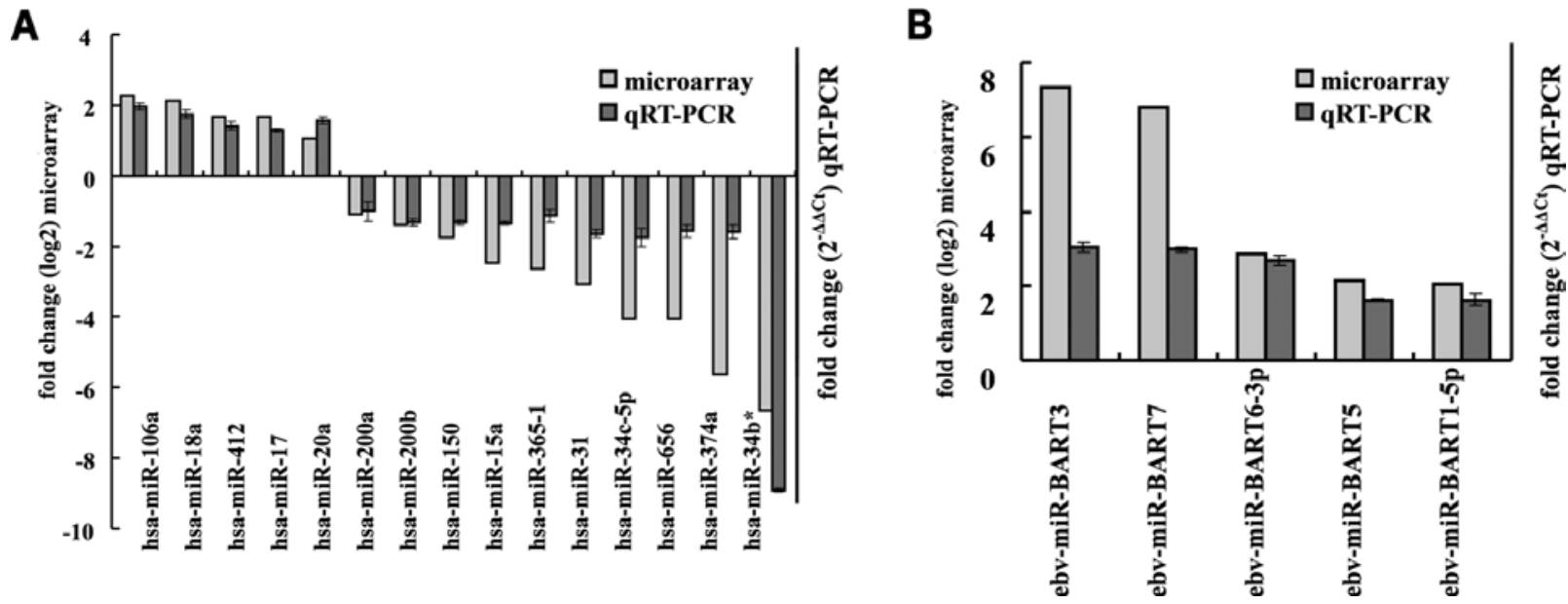

Figure 2. Validation of the microarray-based detection of differentially expressed miRNAs in the NPC tissues and the NNETs using qRT-PCR. Fifteen cellular miRNAs (A) and five EBV mRNAs (B) selected from the microarray data were analyzed using qRT-PCR. The fold-changes in the microarray are presented as $\log ^{2}$ values (left y-axis, light grey bars). Fold-changes according to qRT-PCR were measured using the $2^{-\Delta \Delta C t}$ method and were normalized to the endogenous control U6 (right y-axis, dark grey bars). Error bars represent the standard deviation of the mean (SD). Importantly, the fold-changes (y-axis) cannot be directly compared between assays due to the different calculation methods used. Nevertheless, the general trend of upregulation and downregulation can be compared. NPC, nasopharyngeal carcinoma; NNETs, normal nasopharyngeal epithelial tissues; EBV, Epstein-Barr virus.

described in the Materials and methods. This identified 18,993 host target genes (data not shown), among which 636 were regulated by 4 or more EBV miRNAs (data not shown).

The putative viral target genes of the 9 differentially expressed EBV miRNAs were predicted using vHoT database. This revealed 68 target genes within the EBV genome for 8 differentially expressed EBV miRNAs using any of the three algorithms. Among these, 37 viral target genes were predicted at least by two algorithms (Table IV). Notably, there were no predicted results for ebv-miR-BART18-3p.

Gene Ontology (GO) function and KEGG pathway analyses of the miRNA target genes. A total of 218 and 337 target genes whose expression was regulated by upregulated and downregulated cellular miRNAs, respectively, and negatively correlated with the expression of miRNAs in NPC were uploaded into DAVID for functional and pathway enrichment analyses. The results showed that 118 and $447 \mathrm{GO}$ functions were annotated for upregulated and downregulated cellular miRNA targets $(\mathrm{P} \leq 0.05)$, respectively. Of these, 2 and 35 pathways were enriched statistically $(\mathrm{P} \leq 0.05)$ among the upregulated and the downregulated cellular miRNA targets, respectively.

In addition, the 636 targets that were regulated by 4 or more EBV miRNAs were also uploaded into DAVID for functional and pathway enrichment analyses. Data revealed that $101 \mathrm{GO}$ functions and 12 pathways were statistically enriched $(\mathrm{P} \leq 0.05)$. The most enriched GO terms and KEGG pathways of the target genes are shown in Table V, most of which are involved in carcinogenesis. Notably, the novel differential miRNAs were all enriched in major cellular functions associated with 
Table IV. The 37 putative viral target genes of the 8 upregulated EBV miRNAs predicted by at least two of the algorithms TargetScan, miRanda and PITA from the vHoT database.

\begin{tabular}{|c|c|c|c|}
\hline Category & Target gene & EBV miRNAs & Gene description \\
\hline Capsid & BBRF1 & $\begin{array}{l}\text { ebv-miR-BART10, } \\
\text { ebv-miR-BART1-5p, } \\
\text { ebv-miR-BART4, } \\
\text { ebv-miR-BART8* }\end{array}$ & $\begin{array}{l}\text { Transcription factor, } \\
\text { portal protein }\end{array}$ \\
\hline Capsid & BcLF1 & $\begin{array}{l}\text { ebv-miR-BART1-5p, } \\
\text { ebv-miR-BART3, } \\
\text { ebv-miR-BART4 }\end{array}$ & MCP, VCA, viral lytic gene (late) \\
\hline Capsid & BDLF1 & $\begin{array}{l}\text { ebv-miR-BART7, } \\
\text { ebv-miR-BART3, } \\
\text { ebv-miR-BART5, } \\
\text { ebv-miR-BART1-5p }\end{array}$ & $\mathrm{MCP}$ \\
\hline Capsid & BTRF1 & $\begin{array}{l}\text { ebv-miR-BART10, } \\
\text { ebv-miR-BART7, } \\
\text { ebv-miR-BART6-3p }\end{array}$ & Capsid \\
\hline Capsid & BVRF2 & $\begin{array}{l}\text { ebv-miR-BART6-3p, } \\
\text { ebv-miR-BART3, } \\
\text { ebv-miR-BART8* }\end{array}$ & Maturational protease \\
\hline Capsid & BdRF1 & $\begin{array}{l}\text { ebv-miR-BART3, } \\
\text { ebv-miR-BART4, } \\
\text { ebv-miR-BART5 }\end{array}$ & $\begin{array}{l}\text { Packaging, terminase small subunit, } \\
\text { internal scaffold protein }\end{array}$ \\
\hline Glycoprotein & BALF4 & $\begin{array}{l}\text { ebv-miR-BART3, } \\
\text { ebv-miR-BART1-5p, } \\
\text { ebv-miR-BART7 }\end{array}$ & $\begin{array}{l}\text { Glycoprotein-encoding genes, } \\
\text { product } \mathrm{gB}\end{array}$ \\
\hline Glycoprotein & BDLF3 & $\begin{array}{l}\text { ebv-miR-BART10, } \\
\text { ebv-miR-BART1-5p, } \\
\text { ebv-miR-BART3 }\end{array}$ & $\begin{array}{l}\text { Glycoprotein-encoding genes, } \\
\text { product gp } 150\end{array}$ \\
\hline Glycoprotein & BILF1 & $\begin{array}{l}\text { ebv-miR-BART4, } \\
\text { ebv-miR-BART10, } \\
\text { ebv-miR-BART1-5p, } \\
\text { ebv-miR-BART5, } \\
\text { ebv-miR-BART7 }\end{array}$ & $\begin{array}{l}\text { Product gp64, undetected } \\
\text { in mature virus }\end{array}$ \\
\hline Glycoprotein & BILF2 & $\begin{array}{l}\text { ebv-miR-BART3, } \\
\text { ebv-miR-BART5, } \\
\text { ebv-miR-BART6-3p, } \\
\text { ebv-miR-BART7 }\end{array}$ & Product gp78 \\
\hline Glycoprotein & BLLF1 & $\begin{array}{l}\text { ebv-miR-BART3, } \\
\text { ebv-miR-BART1-5p, } \\
\text { ebv-miR-BART7 }\end{array}$ & $\begin{array}{l}\text { Glycoprotein-encoding genes, } \\
\text { product gp } 350\end{array}$ \\
\hline Glycoprotein & BXLF2 & $\begin{array}{l}\text { ebv-miR-BART1-5p, } \\
\text { ebv-miR-BART4, } \\
\text { ebv-miR-BART7 }\end{array}$ & Product $\mathrm{gH}$ \\
\hline Latent & EBNA-1 & $\begin{array}{l}\text { ebv-miR-BART1-5p, } \\
\text { ebv-miR-BART4, } \\
\text { ebv-miR-BART3, } \\
\text { ebv-miR-BART6-3p, } \\
\text { ebv-miR-BART10, } \\
\text { ebv-miR-BART7, } \\
\text { ebv-miR-BART8* }\end{array}$ & EBNA-1 \\
\hline
\end{tabular}


Table IV. Continued.

\begin{tabular}{|c|c|c|c|}
\hline Category & Target gene & EBV miRNAs & Gene description \\
\hline Latent & EBNA-LP & $\begin{array}{l}\text { ebv-miR-BART5, } \\
\text { ebv-miR-BART6-3p, } \\
\text { ebv-miR-BART1-5p }\end{array}$ & \\
\hline Latent & LMP-1 & $\begin{array}{l}\text { ebv-miR-BART10, } \\
\text { ebv-miR-BART8* }\end{array}$ & LMP-1 \\
\hline Latent & LMP-2A & $\begin{array}{l}\text { ebv-miR-BART1-5p, } \\
\text { ebv-miR-BART4, } \\
\text { ebv-miR-BART10, } \\
\text { ebv-miR-BART5 }\end{array}$ & LMP-2A \\
\hline Latent & LMP-2B & $\begin{array}{l}\text { ebv-miR-BART8*, } \\
\text { ebv-miR-BART1-5p, } \\
\text { ebv-miR-BART7 }\end{array}$ & \\
\hline Latent & RPMS1 & $\begin{array}{l}\text { ebv-miR-BART1-5p, } \\
\text { ebv-miR-BART3, } \\
\text { ebv-miR-BART4, } \\
\text { ebv-miR-BART6-3p, } \\
\text { ebv-miR-BART7, } \\
\text { ebv-miR-BART8*, } \\
\text { ebv-miR-BART10, } \\
\text { ebv-miR-BART5 }\end{array}$ & \\
\hline Replication & BALF5 & $\begin{array}{l}\text { ebv-miR-BART10, } \\
\text { ebv-miR-BART1-5p, } \\
\text { ebv-miR-BART4, } \\
\text { ebv-miR-BART6-3p, } \\
\text { ebv-miR-BART7 }\end{array}$ & Polymerase \\
\hline Replication & BBLF2/BBLF3 & ebv-miR-BART6-3p & Primase-associated factor \\
\hline Tegument & BALF2 & $\begin{array}{l}\text { ebv-miR-BART6-3p, } \\
\text { ebv-miR-BART1-5p, } \\
\text { ebv-miR-BART3, } \\
\text { ebv-miR-BART4, } \\
\text { ebv-miR-BART5, } \\
\text { ebv-miR-BART8* }\end{array}$ & $\begin{array}{l}\text { Replication, viral lytic gene (EA), } \\
\text { ssDNABP }\end{array}$ \\
\hline Tegument & BFRF1A & ebv-miR-BART7 & $\begin{array}{l}\text { Form the terminase complex, } \\
\text { cleavage of DNA concatemers }\end{array}$ \\
\hline Tegument & BGLF1 & $\begin{array}{l}\text { ebv-miR-BART3, } \\
\text { ebv-miR-BART5, } \\
\text { ebv-miR-BART7 }\end{array}$ & Capsid-assoc, packaging \\
\hline Tegument & BGLF3 & $\begin{array}{l}\text { ebv-miR-BART5, } \\
\text { ebv-miR-BART10, } \\
\text { ebv-miR-BART4 }\end{array}$ & Undetected in mature virus \\
\hline Tegument & BNRF1 & $\begin{array}{l}\text { ebv-miR-BART3, } \\
\text { ebv-miR-BART5, } \\
\text { ebv-miR-BART7, } \\
\text { ebv-miR-BART4 }\end{array}$ & MTP \\
\hline Tegument & BOLF1 & $\begin{array}{l}\text { ebv-miR-BART7, } \\
\text { ebv-miR-BART3, } \\
\text { ebv-miR-BART5, } \\
\text { ebv-miR-BART6-3p }\end{array}$ & LTPBP \\
\hline
\end{tabular}


Table IV. Continued.

\begin{tabular}{|c|c|c|c|}
\hline Category & Target gene & EBV miRNAs & Gene description \\
\hline Tegument & BORF2 & $\begin{array}{l}\text { ebv-miR-BART7, } \\
\text { ebv-miR-BART3, } \\
\text { ebv-miR-BART5, } \\
\text { ebv-miR-BART8* }\end{array}$ & $\begin{array}{l}\text { Ribonucleotide reductase } \\
\text { large subunit, RNR-L }\end{array}$ \\
\hline Tegument & BPLF1 & $\begin{array}{l}\text { ebv-miR-BART3, } \\
\text { ebv-miR-BART6-3p, } \\
\text { ebv-miR-BART7, } \\
\text { ebv-miR-BART8*, } \\
\text { ebv-miR-BART10, } \\
\text { ebv-miR-BART1-5p, } \\
\text { ebv-miR-BART4, } \\
\text { ebv-miR-BART5 }\end{array}$ & LTP \\
\hline Tegument & BRLF1 & $\begin{array}{l}\text { ebv-miR-BART5, } \\
\text { ebv-miR-BART7 }\end{array}$ & Product Rta \\
\hline Tegument & BRRF2 & $\begin{array}{l}\text { ebv-miR-BART5, } \\
\text { ebv-miR-BART6-3p }\end{array}$ & $\begin{array}{l}\text { Likely undergoes post-translational } \\
\text { modification, including phosphorylation }\end{array}$ \\
\hline Tegument & BSLF1 & $\begin{array}{l}\text { ebv-miR-BART7, } \\
\text { ebv-miR-BART8* }\end{array}$ & $\begin{array}{l}\text { Virally encoded replication proteins, } \\
\text { primase }\end{array}$ \\
\hline Tegument & BVRF1 & $\begin{array}{l}\text { ebv-miR-BART6-3p, } \\
\text { ebv-miR-BART1-5p, } \\
\text { ebv-miR-BART4 }\end{array}$ & Capsid-assoc, portal plug \\
\hline Transcription factor & BHRF1 & $\begin{array}{l}\text { ebv-miR-BART3, } \\
\text { ebv-miR-BART8* }\end{array}$ & Bcl-2 homolog \\
\hline Transcription factor & BZLF1 & $\begin{array}{l}\text { ebv-miR-BART10, } \\
\text { ebv-miR-BART3, } \\
\text { ebv-miR-BART4 }\end{array}$ & $\begin{array}{l}\text { Zta, viral lytic genes (immediate early, ZEBRA) } \\
\text { site-specific DNA binding protein, } \\
\text { transcriptional activator, origin binding protein, } \\
\text { A BZLF1 mutation affects global expression } \\
\text { of EBV lytic genes }\end{array}$ \\
\hline$?$ & BDLF4 & $\begin{array}{l}\text { ebv-miR-BART4, } \\
\text { ebv-miR-BART8* }\end{array}$ & \\
\hline$?$ & BLLF2 & ebv-miR-BART3 & \\
\hline$?$ & BWRF1 & ebv-miR-BART6-3p & \\
\hline
\end{tabular}

EA, early antigen; EBV, Epstein-Barr virus; LTP, large tegument protein; LTPBP, LTP-binding protein; MCP, major capsid protein; MTP, major tegument protein; ssDNABP, single-stranded DNA-binding protein; VCA, viral capsid antigen.

tumorigenesis, including the regulation of apoptosis, cell proliferation, the cell cycle and cell differentiation (data not shown).

The targets of the upregulated miRNAs were significantly enriched in two pathway annotations $(\mathrm{P}<0.05)$ : the p53 signaling and the cancer pathways. Among the targets of the downregulated miRNAs, the most statistically significant pathway annotations $(\mathrm{P}<0.05)$ were cancer pathways, focal adhesion, the ErbB signaling and the MAPK signaling pathways. Notably, target genes of both the upregulated and downregulated cellular miRNAs are involved in the p53 signaling pathway. The target genes of EBV miRNAs are involved in both the TGF- $\beta$ and Wnt signaling pathways. This suggests that these pathways may play important roles in the development of NPC.

Construction of a cellular miRNA/gene regulatory network. An miRNA/gene regulatory network was constructed using miRNA-target gene pairs. In this network, all target genes were co-regulated by 4 or more miRNAs, enriched in signaling pathways and their expression was negatively correlated with cellular miRNA expression. Among the upregulated miRNAs, 15 miRNAs and 13 target genes were included in a canonical miRNA/gene regulatory network (Fig. 3A); for the downregulated miRNAs, 27 miRNAs and 36 target genes were included (Fig. 3B). 

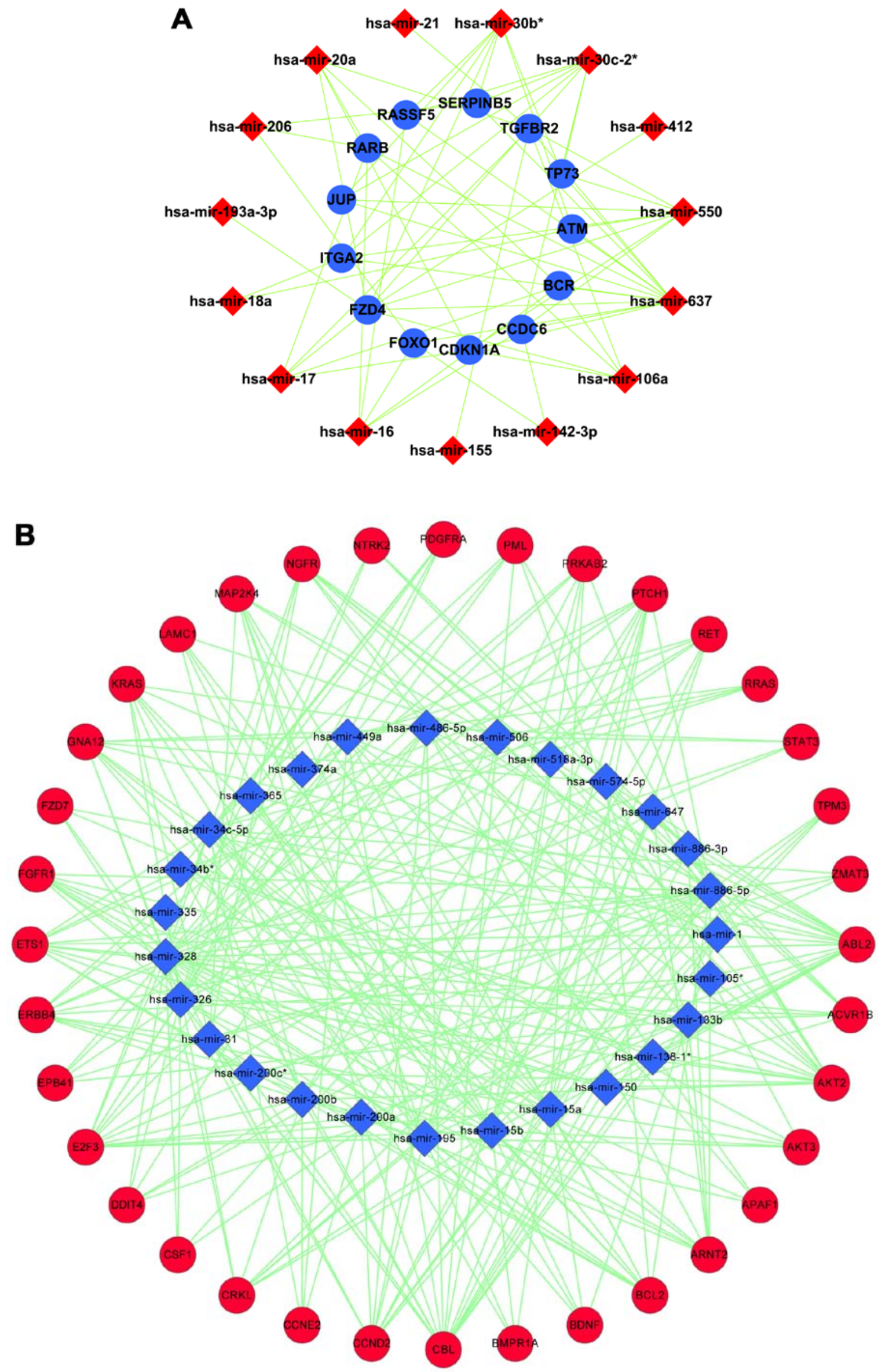

Figure 3. Cellular miRNA/gene regulatory network and intercross regulatory network of cellular and EBV miRNAs in NPC tissues. (A) Fifteen upregulated miRNAs and 13 downregulated target genes were built into a bipartite network using Cytoscape 2.8.3. (B) Twenty-seven downregulated miRNAs and 36 upregulated target genes were built into a bipartite network using Cytoscape 2.8.3. 


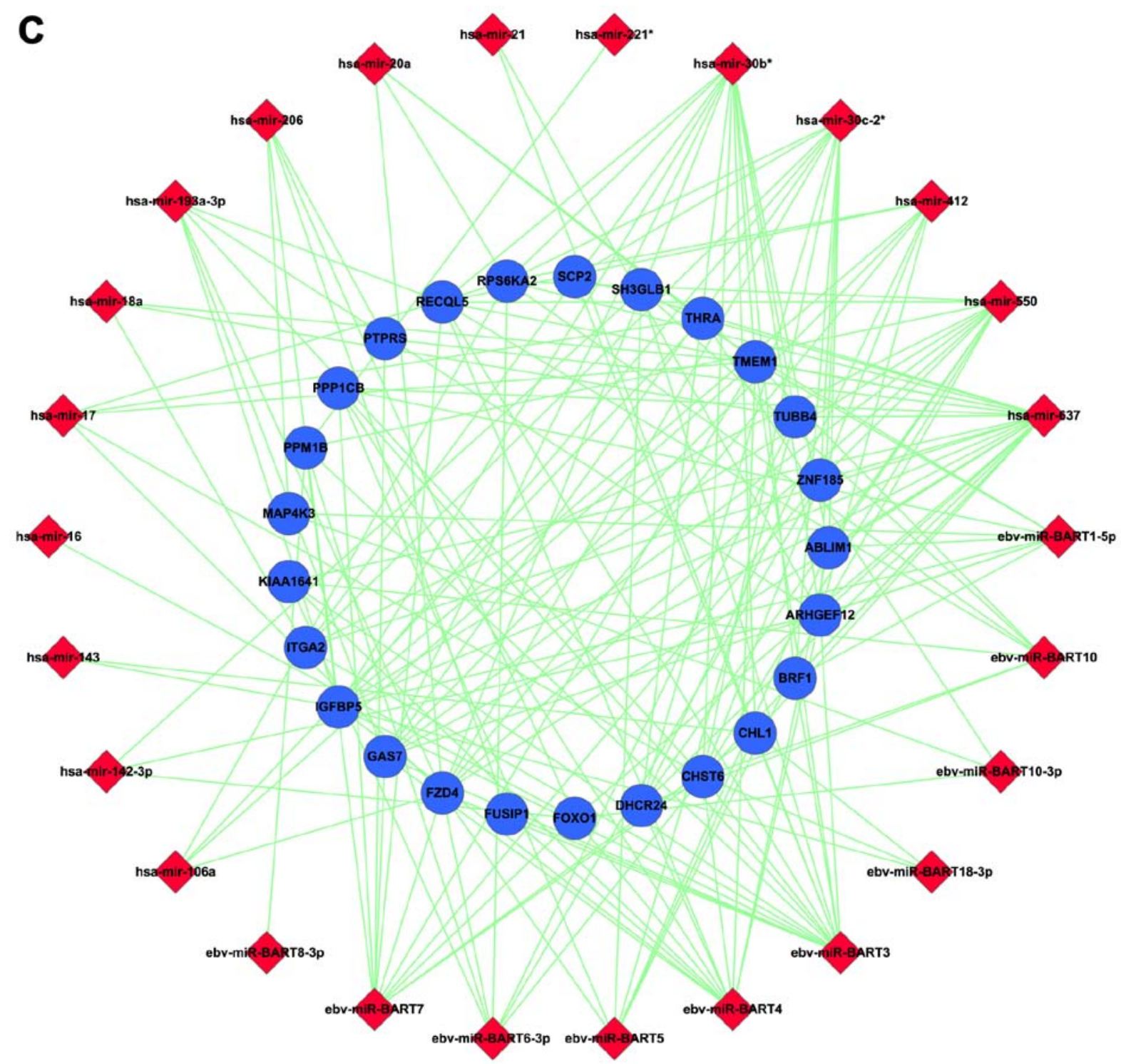

Figure 3. Continued. (C) Twenty-five target genes that were co-regulated by cellular and viral miRNAs and whose expression was negatively correlated with cellular and viral miRNA expression were built into a bipartite network using Cytoscape 2.8.3. The diamonds and ellipses represent the miRNAs and genes, respectively. The red and blue colors represent the relative high and low expression, respectively. NPC, nasopharyngeal carcinoma; EBV, Epstein-Barr virus.

Establishing an intercross regulatory network of cellular and EBV miRNAs in NPC. Analyses identified 25 target genes that were co-regulated by cellular and viral miRNAs and whose expression was negatively correlated with that of the cellular and viral miRNAs. As a result, we established a cellular miRNA/viral miRNA/gene regulatory network in NPC tissues (Fig. 3C). This network combined with the GO and pathway analyses suggests that cellular and EBV miRNAs may inhibit cell apoptosis and promote cell proliferation by jointly regulating the expression of ARHGEF12, SH3GLB1, DHCR24, IGFBP5, ITGA2, FOXO1 and FZD4.

\section{Discussion}

Although the biological effects of many miRNAs have been individually characterized, the effect of the dysregulation of multiple miRNAs on cellular functions and in tumors remains largely unknown. Furthermore, although each miRNA regulates hundreds of genes, their effect on an individual gene is moderate at best $(45,46)$. Recent studies suggested that multiple miRNAs works in concert to regulate targets in a common pathway $(47,48)$. Therefore, pathway analysis rather than individual target gene characterization, provides a better solution for evaluating the biological consequences of global miRNA dysregulation.

Extensive miRNA profiling has become an important tool for elucidating their roles in cancer as well as for identifying biomarkers. To link miRNA profiling data with biological functions a strategy was developed that analyzes the functions and pathways that are regulated collectively by co-expressed miRNAs using computationally predicted targets (49). However, since all target prediction algorithms generate a certain number of false positives, these results significantly reduce the reliability of the data when the targets predicted for multiple miRNAs are combined to analyze functional pathways. Therefore, the results of the pathway enrichment analysis according to co-regulated targets provide a good insight into the functional role of dysregulated miRNAs. 
Table V. GO functional annotation and KEGG pathway analysis of the target genes regulated by the upregulated and downregulated cellular miRNAs and EBV miRNAs in the NPC biopsies.

Term
GO functional annotation of upregulated cellular miRNA target genes
GO:0043065-positive regulation of apoptosis

GO:0006917-induction of apoptosis

GO:0008285-negative regulation of cell proliferation

GO:0006915-apoptosis

GO:0042981-regulation of apoptosis

GO:0007242-intracellular signaling cascade

GO:0042127-regulation of cell proliferation

GO:0019220-regulation of phosphate metabolic process

GO:0006959-humoral immune response

GO:0042744-hydrogen peroxide catabolic process

GO:0045859-regulation of protein kinase activity

GO:0002252-immune effector process

GO:0007264-small GTPase mediated signal transduction

GO:0043066-negative regulation of apoptosis

GO:0034599-cellular response to oxidative stress

GO functional annotation of downregulated cellular miRNA target genes

GO:0042127-regulation of cell proliferation

GO:0008284-positive regulation of cell proliferation

GO:0043067-regulation of programmed cell death

GO:0051726-regulation of cell cycle

GO:0045449-regulation of transcription

GO:0007242-intracellular signaling cascade

GO:0010604-positive regulation of macromolecule

metabolic process

GO:0006357-regulation of transcription from

RNA polymerase II promoter

GO:0007264-small GTPase mediated signal transduction

GO:0043065-positive regulation of apoptosis

GO:0007049-cell cycle

GO:0045596-negative regulation of cell differentiation

GO:0006355-regulation of transcription, DNA-dependent

GO:0009890-negative regulation of biosynthetic process

GO:0045786-negative regulation of cell cycle

GO functional annotation of targets regulated by four or more EBV miRNAs

GO:0010557-positive regulation of macromolecule

biosynthetic process

GO:0010628-positive regulation of gene expression

GO:0045941-positive regulation of transcription

GO:0051254-positive regulation of RNA metabolic process

GO:0006350-transcription

GO:0048598-embryonic morphogenesis

GO:0045449-regulation of transcription

GO:0051173-positive regulation of nitrogen compound

metabolic process

GO:0010604-positive regulation of macromolecule

metabolic process

GO:0010828-positive regulation of glucose transport

GO:0010906-regulation of glucose metabolic process

GO:0035295-tube development

$$
\text { Count }
$$

$\%$

P-value

FDR

(1)

20

0.74

2.78E-06

$1.64 \mathrm{E}-05$

$1.71 \mathrm{E}-05$

$\begin{array}{ll}17 & 0.63\end{array}$

3.08E-05

3.56E-05

26

0.82

$3.56 \mathrm{E}-05$
$1.29 \mathrm{E}-04$

$4.85 \mathrm{E}-04$

$5.26 \mathrm{E}-04$

5.31E-04

$1.25 \mathrm{E}-03$

$1.70 \mathrm{E}-03$

$1.72 \mathrm{E}-03$

$1.98 \mathrm{E}-03$

2.10E-03

2.21E-03

4.62E-03

$2.74 \mathrm{E}-02$

2.84E-02

5.13E-02

5.92E-02

2.15E-01

8.04E-01

$8.72 \mathrm{E}-01$

$8.80 \mathrm{E}-01$

$2.05 \mathrm{E}+00$

$2.78 \mathrm{E}+00$

$2.83 \mathrm{E}+00$

$3.25 \mathrm{E}+00$

$3.44 \mathrm{E}+00$

$3.61 \mathrm{E}+00$

64

38

54

32

104

104
63

63
49

0.19

2.21E-03

2.69E-16

7.99E-10

$1.75 \mathrm{E}-09$

2.16E-08

0.73

2.38

1.44

1.12

1.53E-19

4.54E-13

9.94E-13

1.23E-11

3. $94 \mathrm{E}-10$

6.92E-07

$1.28 \mathrm{E}-09$

2.26E-06

2.53E-09

4.46E-06

44

1.01

3.83E-09

6.73E-06

0.62

4.49E-09

7.90E-06

32

0.73

8.02E-09

$1.41 \mathrm{E}-05$

45

1.03

8.86E-09

$1.56 \mathrm{E}-05$

22

0.50

$1.49 \mathrm{E}-08$

2.62E-05

76

1.74

$1.62 \mathrm{E}-08$

2.84E-05

0.85

$1.76 \mathrm{E}-08$

3.09E-05

14

0.32

2.45E-08

4.30E-05

44

0.73

1.43E-05

2.47E-02

40

0.66

2.30E-05

3.99E-02

39

0.64

2.71E-05

4.70E-02

35

0.58

2.82E-05

4.90E-02

103

1.70

$2.98 \mathrm{E}-05$

5.16E-02

26

0.43

3.39E-05

5.87E-02

121

40

2.00

$5.08 \mathrm{E}-05$

8.80E-02

2.05E-04

$3.56 \mathrm{E}-01$

48

0.79

4.63E-04

8.00E-01

6

0.10

6.50E-04

$1.12 \mathrm{E}+00$

0.12

9.26E-04

$1.59 \mathrm{E}+00$

18

0.30

$1.08 \mathrm{E}-03$

$1.86 \mathrm{E}+00$ 
Table V. Continued.

\begin{tabular}{|c|c|c|c|c|}
\hline Term & Count & $\%$ & P-value & FDR \\
\hline GO:0007268-synaptic transmission & 21 & 0.35 & $2.30 \mathrm{E}-03$ & $3.91 \mathrm{E}+00$ \\
\hline GO:0007167-enzyme linked receptor protein signaling pathway & 23 & 0.38 & $2.36 \mathrm{E}-03$ & $4.02 \mathrm{E}+00$ \\
\hline $\begin{array}{l}\text { GO:0007169-transmembrane receptor protein tyrosine kinase } \\
\text { signaling pathway }\end{array}$ & 17 & 0.28 & $3.32 \mathrm{E}-03$ & $5.61 \mathrm{E}+00$ \\
\hline \multicolumn{5}{|l|}{ KEGG pathways of upregulated cellular miRNA target genes } \\
\hline hsa04115: p53 signaling pathway & 7 & 0.26 & $8.20 \mathrm{E}-04$ & $9.03 \mathrm{E}-01$ \\
\hline hsa05200: pathways in cancer & 13 & 0.48 & $7.10 \mathrm{E}-03$ & $7.58 \mathrm{E}+00$ \\
\hline \multicolumn{5}{|l|}{ KEGG pathways of downregulated cellular miRNA target genes } \\
\hline hsa05200: pathways in cancer & 42 & 0.96 & $9.06 \mathrm{E}-15$ & $1.04 \mathrm{E}-11$ \\
\hline hsa04510: focal adhesion & 24 & 0.55 & $7.78 \mathrm{E}-08$ & $8.91 \mathrm{E}-05$ \\
\hline hsa04012: ErbB signaling pathway & 13 & 0.30 & $1.84 \mathrm{E}-05$ & $2.11 \mathrm{E}-02$ \\
\hline hsa04010: MAPK signaling pathway & 23 & 0.53 & $3.76 \mathrm{E}-05$ & $4.30 \mathrm{E}-02$ \\
\hline hsa04722: neurotrophin signaling pathway & 15 & 0.34 & $3.77 \mathrm{E}-05$ & 4.31E-02 \\
\hline hsa04512: ECM-receptor interaction & 12 & 0.27 & $6.78 \mathrm{E}-05$ & 7.76E-02 \\
\hline hsa04115: p53 signaling pathway & 10 & 0.23 & $2.91 \mathrm{E}-04$ & 3.33E-01 \\
\hline hsa04530:tight junction & 14 & 0.32 & $3.38 \mathrm{E}-04$ & $3.86 \mathrm{E}-01$ \\
\hline hsa04110: cell cycle & 13 & 0.30 & $6.31 \mathrm{E}-04$ & 7.20E-01 \\
\hline hsa04660: T cell receptor signaling pathway & 12 & 0.27 & $6.45 \mathrm{E}-04$ & 7.36E-01 \\
\hline hsa04062: chemokine signaling pathway & 16 & 0.37 & $9.24 \mathrm{E}-04$ & $1.05 \mathrm{E}+00$ \\
\hline hsa04620: Toll-like receptor signaling pathway & 11 & 0.25 & $1.41 \mathrm{E}-03$ & $1.61 \mathrm{E}+00$ \\
\hline hsa04662: B cell receptor signaling pathway & 9 & 0.21 & $2.65 \mathrm{E}-03$ & $2.99 \mathrm{E}+00$ \\
\hline hsa04664: Fc ع RI signaling pathway & 9 & 0.21 & 3.39E-03 & $3.81 \mathrm{E}+00$ \\
\hline hsa04060: cytokine-cytokine receptor interaction & 18 & 0.41 & 4.29E-03 & $4.80 \mathrm{E}+00$ \\
\hline hsa04630:Jak-STAT signaling pathway & 12 & 0.27 & $1.10 \mathrm{E}-02$ & $1.19 \mathrm{E}+01$ \\
\hline hsa04910: insulin signaling pathway & 11 & 0.25 & $1.14 \mathrm{E}-02$ & $1.22 \mathrm{E}+01$ \\
\hline hsa04210: apoptosis & 8 & 0.18 & $2.15 \mathrm{E}-02$ & $2.20 \mathrm{E}+01$ \\
\hline hsa04150: mTOR signaling pathway & 6 & 0.14 & $2.52 \mathrm{E}-02$ & $2.53 \mathrm{E}+01$ \\
\hline hsa04370: VEGF signaling pathway & 7 & 0.16 & $3.31 \mathrm{E}-02$ & $3.20 \mathrm{E}+01$ \\
\hline \multicolumn{5}{|l|}{ KEGG pathways of the targets regulated by four or more EBV miRNAs } \\
\hline hsa04350: TGF- $\beta$ signaling pathway & 10 & 0.17 & $1.87 \mathrm{E}-03$ & $2.14 \mathrm{E}+00$ \\
\hline hsa04920: adipocytokine signaling pathway & 8 & 0.13 & $5.64 \mathrm{E}-03$ & $6.32 \mathrm{E}+00$ \\
\hline hsa00534: heparan sulfate biosynthesis & 5 & 0.08 & $9.15 \mathrm{E}-03$ & $1.01 \mathrm{E}+01$ \\
\hline hsa04530: tight junction & 11 & 0.18 & $1.12 \mathrm{E}-02$ & $1.22 \mathrm{E}+01$ \\
\hline hsa04910: insulin signaling pathway & 11 & 0.18 & $1.18 \mathrm{E}-02$ & $1.28 \mathrm{E}+01$ \\
\hline hsa04520: adherens junction & 8 & 0.13 & $1.19 \mathrm{E}-02$ & $1.29 \mathrm{E}+01$ \\
\hline hsa04310: Wnt signaling pathway & 11 & 0.18 & $2.42 \mathrm{E}-02$ & $2.46 \mathrm{E}+01$ \\
\hline hsa04514: CAMs & 10 & 0.17 & 2.69E-02 & $2.70 \mathrm{E}+01$ \\
\hline hsa05200: pathways in cancer & 18 & 0.30 & $3.60 \mathrm{E}-02$ & $3.45 \mathrm{E}+01$ \\
\hline
\end{tabular}

CAM, cell adhesion molecules; EBV, Epstein-Barr virus; GO, Gene Ontology; NPC, nasopharyngeal carcinoma; KEGG, Kyoto Encyclopedia of Genes and Genomes; FDR, false discovery rate.

In the present study, we analyzed both the expression profiles of both cellular and EBV miRNAs in pooled laser capture microdissected NPC tissues using microarrays. The analysis of pooled NPC tissues avoids the large variation that occurs between samples to help identify bona fide miRNAs and target genes that are dysregulated in NPCs. In addition, few studies have performed a systematic analysis of the cellular and EBV miRNA expression profiles in 20 NPC tissues. Our analyses identified 50 differentially expressed cellular miRNAs and 9 differentially expressed EBV miRNAs, which regulate a total of 555 and 636 target genes, respectively. Furthermore, DAVID analysis revealed 37 and 12 pathways that were modulated by cellular and EBV miRNAs in NPC, respectively. Among these, the cellular miRNAs mainly regulate pathways involved in apoptosis and cell proliferation, whereas the EBV miRNAs mainly target genes in the TGF- $\beta$ and Wnt signaling pathways. 
Furthermore, we constructed a cellular and EBV miRNA/ target gene regulatory network (Fig. 3). This global miRNA and target gene analysis in NPC tissues is important to further understand the mechanism of NPC carcinogenesis and provide guidance for the diagnosis and treatment.

Previously, different groups identified a number of miRNAs that were dysregulated in NPC tissues using microarrays or RT-PCR, yet there was poor inter-platform consistency $(11,32,33,36)$. Sengupta et al (11) analyzed 31 tumor and 10 normal surrounding tissues using microarrays, and identified 8 cellular miRNAs with $\geq 5$-fold differential expression between tumor and normal tissues. Chen et al (32) quantified the expression of 270 human miRNAs in 13 NPCs and 9 adjacent normal tissues using RT-PCR, and identified 11 and 24 miRNAs that were significantly upregulated and downregulated in NPC samples. Li et al (36) screened the miRNA expression changes in 8 NPCs and 4 normal nasopharyngeal tissues using microarrays, and identified 34 miRNAs that were expressed differentially in NPC tissues. Luo et al (33) performed miRNA microarrays using 20 different stage NPCs and 6 normal samples, and identified 11 miRNAs that may mediate the development of NPC. Among these four studies, only miR-34b and miR-34c were commonly identified as downregulated in NPC tissues. This poor consistency may be partially attributable to variations in NPC samples and the different platforms used for the measurements. Notably, miR-34b and miR-34c were also significantly downregulated in our pooled NPC tissues (Table III). miR-34 is a downstream target of p53, and promotes apoptosis (50). The downregulation of miR-34b and miR-34c strongly suggest that NPC exhibits enhanced cell survival due to inhibition of apoptosis pathways.

As previously mentioned, Sengupta et al (11) analyzed only miRNAs with $\geq 5$-fold differential expression between tumor and normal tissues. They may have excluded some important miRNAs from their dataset. Therefore, we searched for other miRNAs that were commonly dysregulated in the remaining three datasets. This revealed that miR-18a was commonly overexpressed in NPC in these datasets as well as the present study. miR-18a belongs to the miR-17-92 cluster, and enhances tumorigenesis by inducing c-Myc expression (51). Similarly, miR-449a was commonly downregulated in NPC in the current dataset as well as by Chen et al (32) and Li et al (36). In addition, let-7b was downregulated significantly in NPC in the current dataset, which was consistent with the findings of Li et al (36). Since let-7 targets key cell cycle proto-oncogenes such as RAS, CDC25a, CDK6, and cyclin D to repress cell proliferation (52), we speculate that the downregulation of Let-7 in NPC may play a major role in tumor growth. Furthermore, miR-200a, miR-200b, and miR-31 were all downregulated in the present study and in the report by Chen et al (32). The miR-200 family inhibits NPC cell growth, migration and invasion (18). miR-31 is a tumor suppressor gene in breast cancer, and its reduced expression in NPC contributes to tumorigenesis and metastasis (53). Therefore, miR-18a, miR-449a, Let-7, the miR-200 family and miR-31 may be commonly dysregulated in NPC, and play roles in its tumorigenesis, proliferation and metastasis.

GO analysis found that the cellular miRNAs upregulated in NPC mainly regulate apoptosis-related genes, whereas the downregulated miRNAs primarily modulate genes related to cell proliferation. KEGG pathway analysis demonstrated that the upregulated miRNAs mainly target the p53 signaling pathway in cancer, whereas the downregulated miRNAs modulate pathways related to cancer, focal adhesion, ErbB and MAPK signaling (Table V). Chen et al (32) also observed that miRNAs modulated apoptosis and survival pathways and enhanced cell cycle progression and signaling pathways in NPC. Therefore, it is possible that the upregulated miRNAs such as miR-34b and -34c in NPC inhibit p53-mediated apoptosis to enhance cell survival; conversely, the downregulated miRNAs promote cell growth. This may be an important mechanism for the development of NPCs. Furthermore, we constructed a cellular miRNA/gene regulatory network, which provides vital information for the role of miRNAs in the tumorigenesis of NPCs.

EBV miRNAs are very abundant in NPC, and they may play important regulatory roles in tumor progression (31). Previously, Wong et al (39) pooled 5 NPC samples and identified 29 upregulated EBV-encoded miRNAs in NPC tissues. In the present study, the $9 \mathrm{EBV}$ miRNAs that were upregulated in 20 NPC tissues (BART1-5p, BART3, BART4, BART5, BART6, BART7, BART8, BART10 and BART18-3p) were also identified by Wong et al (39). In addition, Zhu et al (38) reported that EBV-miR-BART4, BRAT6, and BRAT7 were expressed significantly in NPC samples using northern blotting. Some of these EBV miRNAs modulate the transformation, growth and survival of NPC. For example, miR-BART3 targets the DICE1 tumor suppressor to promote cellular growth and transformation in NPC (54). In addition, miR-BART5 regulates a cellular protein named p53-upregulated modulator of apoptosis (PUMA) to modulate host cell survival (25). miR-BART7 was proposed as a novel serological biomarker for the diagnosis of NPC (55). In the present study, KEGG pathway analysis showed that the upregulated EBV miRNAs mainly target genes in the TGF- $\beta$ and Wnt signaling pathways. Both the TGF- $\beta$ and Wnt signaling pathways were previously shown to be dysregulated in NPC $(32,56)$. In addition, the TGF- $\beta$ signaling pathway plays a critical role in the progression of human cancer (57). Activation of the Wnt signaling pathway in NPC is also associated with NPC development (58). Therefore, the dysregulation of EBV miRNAs such as BART3 and BART5 in NPC plays key roles in the transformation and growth of NPC.

To globally understand the genesis of NPC, we also established a cellular miRNA/viral miRNA/gene regulatory network in NPC tissues (Fig. 3C). This network may help explain how the EBV works with cellular miRNAs to regulate gene expression and promote the transformation of NPC.

In conclusion, we performed a systematic characterization of the cellular and EBV miRNA expression profiles in NPC tissues. Our data suggest that cellular miRNAs such as miRNA-34b, miRNA-34c, miR-18a and Let-7 mainly target genes involved in apoptosis and cell proliferation. In addition, we found that the upregulated EBV miRNAs in NPCs mainly target the TGF- $\beta$ and Wnt signaling pathways. These identified cellular and EBV miRNAs may be potential therapeutic targets for the treatment of NPC. In addition, we identified 25 target genes that were co-regulated by cellular and viral miRNAs. Further analysis of this cellular miRNA/viral miRNA/gene regulatory network may unveil the mechanism of NPC carcinogenesis. 


\section{Acknowledgements}

The present study was supported by the National Natural Science Foundation of China (nos. 81230053, 81172559 and 81272959), the Hunan Provincial Natural Science Foundation of China (no. 10JJ3045), and the Scientific Research Fund of Hunan Provincial Education Department (no. 08C566).

\section{References}

1. Ambros V: The functions of animal microRNAs. Nature 431: 350-355, 2004.

2. Croce CM and Calin GA: miRNAs, cancer, and stem cell division. Cell 122: 6-7, 2005.

3. Bushati N and Cohen SM: microRNA functions. Annu Rev Cell Dev Biol 23: 175-205, 2007.

4. Chen CZ: MicroRNAs as oncogenes and tumor suppressors. N Engl J Med 353: 1768-1771, 2005.

5. Calin GA, Sevignani C, Dumitru CD, Hyslop T, Noch E, Yendamuri S, Shimizu M, Rattan S, Bullrich F, Negrini M, et al: Human microRNA genes are frequently located at fragile sites and genomic regions involved in cancers. Proc Natl Acad Sci USA 101: 2999-3004, 2004.

6. Lu J, Getz G, Miska EA, Alvarez-Saavedra E, Lamb J, Peck D, Sweet-Cordero A, Ebert BL, Mak RH, Ferrando AA, et al: MicroRNA expression profiles classify human cancers. Nature 435: 834-838, 2005

7. Murakami Y, Yasuda T, Saigo K, Urashima T, Toyoda H, Okanoue $\mathrm{T}$ and Shimotohno K: Comprehensive analysis of microRNA expression patterns in hepatocellular carcinoma and non-tumorous tissues. Oncogene 25: 2537-2545, 2006.

8. Kida Y and Han YP: MicroRNA expression in colon adenocarcinoma. JAMA 299: 2628-2629, 2008.

9. Yanaihara N, Caplen N, Bowman E, Seike M, Kumamoto K, Yi M, Stephens RM, Okamoto A, Yokota J, Tanaka T, et al: Unique microRNA molecular profiles in lung cancer diagnosis and prognosis. Cancer Cell 9: 189-198, 2006.

10. Schetter AJ, Leung SY, Sohn JJ, Zanetti KA, Bowman ED, Yanaihara N, Yuen ST, Chan TL, Kwong DLW, Au GKH, et al: MicroRNA expression profiles associated with prognosis and therapeutic outcome in colon adenocarcinoma. JAMA 299: 425-436, 2008

11. Sengupta S, den Boon JA, Chen IH, Newton MA, Stanhope SA, Cheng YJ, Chen CJ, Hildesheim A, Sugden B and Ahlquist P: MicroRNA $29 \mathrm{c}$ is down-regulated in nasopharyngeal carcinomas, up-regulating mRNAs encoding extracellular matrix proteins. Proc Natl Acad Sci USA 105: 5874-5878, 2008.

12. Alajez NM, Lenarduzzi M, Ito E, Hui AB, Shi W, Bruce J, Yue S, Huang $\mathrm{SH}, \mathrm{Xu} \mathrm{W}$, Waldron J, et al: miR-218 suppresses nasopharyngeal cancer progression through downregulation of survivin and the SLIT2-ROBO1 pathway. Cancer Res 71: 2381-2391, 2011

13. Lu J, He ML, Wang L, Chen Y, Liu X, Dong Q, Chen YC, Peng Y, Yao KT, Kung HF, et al: MiR-26a inhibits cell growth and tumorigenesis of nasopharyngeal carcinoma through repression of EZH2. Cancer Res 71: 225-233, 2011.

14. Yi C, Wang Q, Wang L, Huang Y, Li L, Liu L, Zhou X, Xie G, Kang $\mathrm{T}$, Wang $\mathrm{H}$, et al: miR-663, a microRNA targeting p21 $1_{\text {WAF1/CIP1 }}$, promotes the proliferation and tumorigenesis of nasopharyngeal carcinoma. Oncogene 31: 4421-4433, 2012.

15. Wong TS, Man OY, Tsang CM, Tsao SW, Tsang RK, Chan JY, Ho WK, Wei WI and To VS: MicroRNA let-7 suppresses nasopharyngeal carcinoma cells proliferation through downregulating c-Myc expression. J Cancer Res Clin Oncol 137: 415-422, 2011.

16. Zhang L, Deng T, Li X, Liu H, Zhou H, Ma J, Wu M, Zhou M, Shen S, Li X, et al: microRNA-141 is involved in a nasopharyngeal carcinoma-related genes network. Carcinogenesis 31: 559-566, 2010

17. Deng M, Tang H, Zhou Y, Zhou M, Xiong W, Zheng Y, Ye Q, Zeng X, Liao Q, Guo X, et al: miR-216b suppresses tumor growth and invasion by targeting KRAS in nasopharyngeal carcinoma. J Cell Sci 124: 2997-3005, 2011.

18. Xia H, Ng SS, Jiang S, Cheung WK, Sze J, Bian XW, Kung HF and Lin MC: miR-200a-mediated downregulation of ZEB2 and CTNNB1 differentially inhibits nasopharyngeal carcinoma cell growth, migration and invasion. Biochem Biophys Res Commun 391: 535-541, 2010
19. Busson P, Keryer C, Ooka T and Corbex M: EBV-associated nasopharyngeal carcinomas: From epidemiology to virustargeting strategies. Trends Microbiol 12: 356-360, 2004.

20. Wei WI and Sham JS: Nasopharyngeal carcinoma. Lancet 365: 2041-2054, 2005.

21. Qiu J, Cosmopoulos K, Pegtel M, Hopmans E, Murray P, Middeldorp J, Shapiro M and Thorley-Lawson DA: A novel persistence associated EBV miRNA expression profile is disrupted in neoplasia. PLoS Pathog 7: e1002193, 2011.

22. Barth S, Meister G and Grässer FA: EBV-encoded miRNAs. Biochim Biophys Acta 1809: 631-640, 2011.

23. Xia T, O'Hara A, Araujo I, Barreto J, Carvalho E, Sapucaia JB, Ramos JC, Luz E, Pedroso C, Manrique M, et al: EBV microRNAs in primary lymphomas and targeting of $C X C L-11$ by ebv-mir-BHRF1-3. Cancer Res 68: 1436-1442, 2008.

24. Nachmani D, Stern-Ginossar N, Sarid R and Mandelboim O: Diverse herpesvirus microRNAs target the stress-induced immune ligand MICB to escape recognition by natural killer cells. Cell Host Microbe 5: 376-385, 2009.

25. Choy EY, Siu KL, Kok KH, Lung RW, Tsang CM, To KF, Kwong DL, Tsao SW and Jin DY: An Epstein-Barr virus-encoded microRNA targets PUMA to promote host cell survival. J Exp Med 205: 2551-2560, 2008

26. Li Z, Chen X, Li L, Liu S, Yang L, Ma X, Tang M, Bode AM, Dong Z, Sun L, et al: EBV encoded miR-BHRF1-1 potentiates viral lytic replication by downregulating host $\mathrm{p} 53$ in nasopharyngeal carcinoma. Int J Biochem Cell Biol 44: 275-279, 2012.

27. Lo AK, To KF, Lo KW, Lung RW, Hui JW, Liao G and Hayward SD: Modulation of LMP1 protein expression by EBV-encoded microRNAs. Proc Natl Acad Sci USA 104: 16164-16169, 2007.

28. Barth S, Pfuhl T, Mamiani A, Ehses C, Roemer K, Kremmer E, Jäker C, Höck J, Meister G and Grässer FA: Epstein-Barr virusencoded microRNA miR-BART2 down-regulates the viral DNA polymerase BALF5. Nucleic Acids Res 36: 666-675, 2008.

29. Lung RWM, Tong JHM, Sung YM, Leung PS, Ng DCH, Chau SL, Chan AWH, Ng EKO, Lo KW and To KF: Modulation of LMP2A expression by a newly identified Epstein-Barr virusencoded microRNA miR-BART22. Neoplasia 11: 1174-1184, 2009.

30. Lo AK, Dawson CW, Jin DY and Lo KW: The pathological roles of BART miRNAs in nasopharyngeal carcinoma. J Pathol 227: 392-403, 2012.

31. Marquitz AR and Raab-Traub N: The role of miRNAs and EBV BARTs in NPC. Semin Cancer Biol 22: 166-172, 2012.

32. Chen HC, Chen GH, Chen YH, Liao WL, Liu CY, Chang KP, Chang YS and Chen SJ: MicroRNA deregulation and pathway alterations in nasopharyngeal carcinoma. Br J Cancer 100: 1002-1011, 2009.

33. Luo Z, Zhang L, Li Z, Li X, Li G, Yu H, Jiang C, Dai Y, Guo X, Xiang $\mathrm{J}$, et al: An in silico analysis of dynamic changes in microRNA expression profiles in stepwise development of nasopharyngeal carcinoma. BMC Med Genomics 5: 3, 2012

34. Liu N, Chen NY, Cui RX, Li WF, Li Y, Wei RR, Zhang MY, Sun Y, Huang BJ, Chen M, et al: Prognostic value of a microRNA signature in nasopharyngeal carcinoma: A microRNA expression analysis. Lancet Oncol 13: 633-641, 2012.

35. Zeng X, Xiang J, Wu M, Xiong W, Tang H, Deng M, Li X, Liao Q, Su B, Luo Z, et al: Circulating miR-17, miR-20a, miR-29c, and miR-223 combined as non-invasive biomarkers in nasopharyngeal carcinoma. PLoS One 7: e46367, 2012.

36. Li T, Chen JX, Fu XP, Yang S, Zhang Z, Chen KhH and Li Y: microRNA expression profiling of nasopharyngeal carcinoma. Oncol Rep 25: 1353-1363, 2011.

37. Chen SJ, Chen GH, Chen YH, Liu CY, Chang KP, Chang YS and Chen HC: Characterization of Epstein-Barr virus miRNAome in nasopharyngeal carcinoma by deep sequencing. PLoS One 5: e12745, 2010

38. Zhu JY, Pfuhl T, Motsch N, Barth S, Nicholls J, Grässer F and Meister G: Identification of novel Epstein-Barr virus microRNA genes from nasopharyngeal carcinomas. J Virol 83: 3333-3341, 2009.

39. Wong AM, Kong KL, Tsang JW, Kwong DL and Guan XY: Profiling of Epstein-Barr virus-encoded microRNAs in nasopharyngeal carcinoma reveals potential biomarkers and oncomirs. Cancer 118: 698-710, 2012.

40. Cheng AL, Huang WG, Chen ZC, Peng F, Zhang PF, Li MY, Li F, Li JL, Li C, Yi H, et al: Identification of novel biomarkers for differentiation and prognosis of nasopharyngeal carcinoma by laser capture microdissection and proteomic analysis. Clin Cancer Res 14: 435-445, 2008. 
41. Elefant N, Berger A, Shein H, Hofree M, Margalit H and Altuvia Y: RepTar: A database of predicted cellular targets of host and viral miRNAs. Nucleic Acids Res 39: D188-D194, 2011.

42. Maragkakis M, Reczko M, Simossis VA, Alexiou P, Papadopoulos GL, Dalamagas T, Giannopoulos G, Goumas G, Koukis E, Kourtis K, et al: DIANA-microT web server: Elucidating microRNA functions through target prediction. Nucleic Acids Res 37: W273-W276, 2009.

43. Yang JH, Li JH, Shao P, Zhou H, Chen YQ and Qu LH: starBase: A database for exploring microRNA-mRNA interaction maps from Argonaute CLIP-Seq and Degradome-Seq data. Nucleic Acids Res 39: D202-D209, 2011.

44. Kim H, Park S, Min H and Yoon S: vHoT: A database for predicting interspecies interactions between viral microRNA and host genomes. Arch Virol 157: 497-501, 2012.

45. Baek D, Villén J, Shin C, Camargo FD, Gygi SP and Bartel DP: The impact of microRNAs on protein output. Nature 455: 64-71, 2008.

46. Selbach M, Schwanhäusser B, Thierfelder N, Fang Z, Khanin R and Rajewsky N: Widespread changes in protein synthesis induced by microRNAs. Nature 455: 58-63, 2008.

47. Cloonan N, Brown MK, Steptoe AL, Wani S, Chan WL, Forrest AR, Kolle G, Gabrielli B and Grimmond SM: The miR-17-5p microRNA is a key regulator of the G1/S phase cell cycle transition. Genome Biol 9: R127, 2008.

48. Liu Q, Fu H, Sun F, Zhang H, Tie Y, Zhu J, Xing R, Sun Z and Zheng X: miR-16 family induces cell cycle arrest by regulating multiple cell cycle genes. Nucleic Acids Res 36: 5391-5404, 2008.

49. Gusev Y, Schmittgen TD, Lerner M, Postier R and Brackett D: Computational analysis of biological functions and pathways collectively targeted by co-expressed microRNAs in cancer. BMC Bioinformatics 8 (Suppl 7): S16, 2007.
50. Chang TC, Wentzel EA, Kent OA, Ramachandran K, Mullendore M, Lee KH, Feldmann G, Yamakuchi M, Ferlito M, Lowenstein CJ, et al: Transactivation of miR-34a by p53 broadly influences gene expression and promotes apoptosis. Mol Cell 26: 745-752, 2007.

51. Dews M,Homayouni A, Yu D, Murphy D, Sevignani C, Wentzel E, Furth EE, Lee WM, Enders GH, Mendell JT, et al: Augmentation of tumor angiogenesis by a Myc-activated microRNA cluster. Nat Genet 38: 1060-1065, 2006.

52. Johnson CD, Esquela-Kerscher A, Stefani G, Byrom M, Kelnar K, Ovcharenko D, Wilson M, Wang X, Shelton J, Shingara J, et al: The let-7 microRNA represses cell proliferation pathways in human cells. Cancer Res 67: 7713-7722, 2007.

53. O'Day E and Lal A: MicroRNAs and their target gene networks in breast cancer. Breast Cancer Res 12: 201, 2010.

54. Lei T, Yuen KS, Xu R, Tsao SW, Chen H, Li M, Kok KH and Jin DY: Targeting of DICE1 tumor suppressor by Epstein-Barr virus-encoded miR-BART3* microRNA in nasopharyngeal carcinoma. Int J Cancer 133: 79-87, 2013.

55. Zhang G, Zong J, Lin S, Verhoeven RJ, Tong S, Chen Y, Ji M, Cheng W, Tsao SW, Lung M, et al: Circulating Epstein-Barr virus microRNAs miR-BART7 and miR-BART13 as biomarkers for nasopharyngeal carcinoma diagnosis and treatment. Int $\mathrm{J}$ Cancer 136: E301-E312, 2015.

56. Zeng ZY, Zhou YH, Zhang WL, Xiong W, Fan SQ, Li XL, Luo XM, Wu MH, Yang YX, Huang C, et al: Gene expression profiling of nasopharyngeal carcinoma reveals the abnormally regulated Wnt signaling pathway. Hum Pathol 38: 120-133, 2007.

57. Meulmeester E and Ten Dijke P: The dynamic roles of TGF- $\beta$ in cancer. J Pathol 223: 205-218, 2011.

58. Chou J, Lin YC, Kim J, You L, Xu Z, He B and Jablons DM: Nasopharyngeal carcinoma - review of the molecular mechanisms of tumorigenesis. Head Neck 30: 946-963, 2008. 